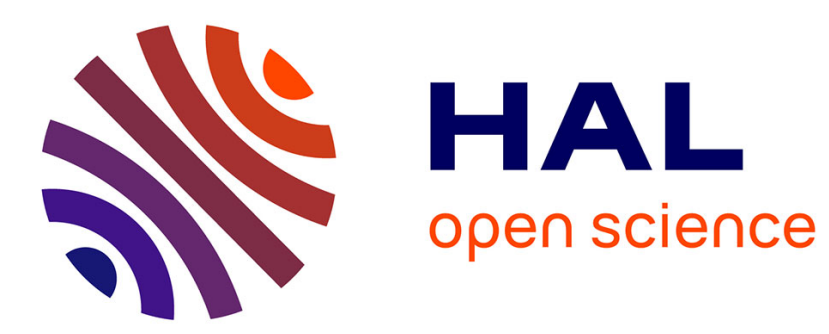

\title{
Role of Transport in the First Atomic Layers of Nanoparticles in Lithium Batteries
}

\author{
Frédéric Lantelme, Jiwei Ma, Damien Dambournet
}

\section{To cite this version:}

Frédéric Lantelme, Jiwei Ma, Damien Dambournet. Role of Transport in the First Atomic Layers of Nanoparticles in Lithium Batteries. Journal of The Electrochemical Society, 2020, 167 (14), pp.140538. 10.1149/1945-7111/abc76b . hal-03060520

\section{HAL Id: hal-03060520 \\ https://hal.sorbonne-universite.fr/hal-03060520}

Submitted on 14 Dec 2020

HAL is a multi-disciplinary open access archive for the deposit and dissemination of scientific research documents, whether they are published or not. The documents may come from teaching and research institutions in France or abroad, or from public or private research centers.
L'archive ouverte pluridisciplinaire HAL, est destinée au dépôt et à la diffusion de documents scientifiques de niveau recherche, publiés ou non, émanant des établissements d'enseignement et de recherche français ou étrangers, des laboratoires publics ou privés. 
3 Frédéric Lantelme $e^{\dagger, \hbar^{*}}$, Jiwei Ma ${ }^{\dagger, \hbar, \times}$, Damien Dambournet ${ }^{\dagger, \hbar}$

4 PHENIX, F-75005 Paris, France. Amiens cedex, France. University, Shanghai 201804, China.

(1)

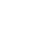

\footnotetext{
* E-mail: frederic.lantelme@upmc.fr
}

\title{
Role of transport in the first atomic layers of the nanoparticles in lithium batteries
}

†Sorbonne Université, CNRS, Physico-chimie des électrolytes et nano-systèmes interfaciaux,

$\$$ Réseau sur le Stockage Electrochimique de l’Energie (RS2E), FR CNRS 3459, 80039

${ }^{\times}$Institute of New Energy for Vehicles, School of Materials Science and Engineering, Tongji

\begin{abstract}
A theoretical approach of the insertion/deinsertion of lithium in host materials is proposed to take into account some phenomena not included in classical analyses of lithium battery operation. This is mainly related to the variation of the transport properties in the first atomic layers in the vicinity of the interface which can give rise to rapid storage phenomena. These processes are of great importance in the case of electrodes made of nanoparticles as a result of the considerable development of the interfacial surface. For example, they can be at the origin of the important capacitive behavior of the system. A model based on the numerical integration of transport equations is presented to describe these complex mechanisms. The theoretical analysis is illustrated on the model case of lithium insertion in electrodes made of nanoparticles of anatase and fluorinated anatase.
\end{abstract}




\section{Introduction}

The pressing need for transportable electrical energy sources has given rise to a great deal of work in recent decades. Among these, lithium-based batteries occupy a prominent place due to the electrochemical properties of this element joined to its low density. This sector has benefited from intensive research which has improved performance ${ }^{1-3}$. A large part of the research was devoted to the development of electrode materials which exhibit a suitable free energy of formation and thus a high open circuit voltage for a high performance battery. In order to improve the battery power, they also should allow a rapid transport of lithium atoms. A device commonly used to fulfill these criteria is based on the lithium insertion in a host material, generally a metal oxide such as $\mathrm{V}_{2} \mathrm{O}_{5}, \mathrm{Nb}_{2} \mathrm{O}_{5}, \mathrm{LiCoO}_{2}, \mathrm{TiO}_{2}$, etc ${ }^{4}$.

50

However, a weakness of the system comes from the very slow transport of lithium atoms in the crystal lattice of the host material. An effective way to overcome this difficulty is to reduce the size of host particles which reduces the diffusion length and promotes faster transport ${ }^{5}$. Hence the idea of using particles of reactive material at a nanometric dimension, which considerably increases the contact surface and facilitates the lithium storage; an issue of nanoparticles is accelerated side reactions. The study of these systems has given rise to a large number of research work which focus on the nature of the host material, its preparation and the properties of lithium intercalation. Various systems have been developed which are essentially characterized by the nature of the host material in order to obtain the best lithium intercalation qualities. This work has led to a lot of research to get a more accurate view of the reaction mechanisms. Here is a brief overview of the main work in this area.

The insertion of Li-ions within cathode materials during the discharging of a battery oftentimes brings about one or more structural transformations ${ }^{6}$. A theoretical investigation of the effects of elastic coherency strain on the thermodynamics, kinetics, and morphology of intercalation in single $\mathrm{LiFePO}_{4}$ nanoparticles yields new insights into this important battery material ${ }^{7}$. The kinetics of a two-phase electrochemical reaction in $\mathrm{Li}_{x} \mathrm{FePO}_{4}$ indicates that the phase transition proceeds with a one-dimensional phase-boundary movement ${ }^{8}$. The core-shell, the 
compositional striping modulations and the resulting strain gradients point to the need to design cathode materials and electrode architectures to mitigate such pronounced local inhomogeneities in Li-ion intercalation and diffusion ${ }^{9}$.

The different specific constraints of the batteries have given rise to many analytical descriptions of transport phenomena from conventional models. For example, concentration dependent diffusion coefficients are found to be necessary to match experimental results ${ }^{10}$. On the other hand, in a finer way, it was suggested to represent the phase transitions as moving boundary points where jump discontinuities in concentration occur; the question of when it is necessary to include these effects in a transport model is difficult to resolve, because the measured values of the open-circuit voltage for real materials are never completely flat and always exhibit a small dependence on state of charge; it was shown that the jump discontinuities arise as a singular limit of a nonlinear diffusion equation when chemical potentials are used as the driving force for transport ${ }^{11}$. It was also pointed out that variations in the chemical diffusivities over a significant range of lithiation are shown to be explained by the inclusion of both the thermodynamic factor and the flux of lithium due to bulk motion of material ${ }^{12,13}$.

In addition to the influence of the thermodynamic properties and of the structural changes of the materials described above, it is important to pay our attention to an essential characteristic of the batteries, namely the nanometric dimension of the active particles. As already pointed out, the use of nanoparticles has the advantage of significantly increasing the electroactive surface. The increase in surface area is generally taken into account by the introduction of a roughness factor in the calculation elements ${ }^{14,15}$. But this correction does not take into account the specific nature of the transport in the very first atomic layers of the surface thus introduced. Indeed, another consequence of the use of nanoparticles is to considerably shorten the path of lithium transport, which is now reduced to distances of the order of atomic dimensions. Attention should be drawn to the fact that much of the transport is no longer carried out in the compact layers but remains confined to the first atomic layers of the substrate. In the superficial region the transport properties are greatly affected by the loosening of the surface structure of the material. This phenomenon is generally negligible for large conventional systems but becomes predominant when transport does not exceed a few atomic layers.

The purpose of this paper is to analyze and highlight the important role of transport phenomena in the surface layers of the substrate material: given the nanometric nature of the particles, the 
influence of transport kinetics in these few superficial atomic layers becomes significant. To tackle in a more structural way the origin of these phenomena it seems interesting to take inspiration from already developed models dealing with the analysis of the transport kinetics in the first atomic layers of the host material ${ }^{16}$.

After a brief description of the materials and of the experimental setup, we will describe the elementary principles involved in the reaction mechanisms. Particular emphasis will be placed on deviations from ideality, on the variation of transport properties in the surface layers, and on the formation of storage layers at the interface; then, to interpret the modifications thus introduced we will briefly recall the principle of the integration of basic equations by the finite difference method ${ }^{17,18}$. Finally, the investigations will be illustrated on the model case of lithium insertion in anatase or fluorinated anatase crystallites.

\section{Experimental}

Synthesis. The experimental study examined nanoparticles of anatase and fluorinated anatase; in fact, the action of a fluorinated agent improves the storage properties of the battery. The materials were synthesized using the sol-gel chemistry without and with a fluorinating agent, as described in a previous report ${ }^{19}$. The degree of fluorination may vary depending on the procedure employed; we used a compound with $22 \%$ cationic vacancies, corresponding to $\operatorname{Ti}_{0.78} \square_{0.22} \mathrm{O}_{1.12} \mathrm{~F}_{0.40}(\mathrm{OH})_{0.48}$, where $\square$ represents a cationic vacancy. For more details concerning this formulation see ref. (19).

Electrochemistry. Electrochemical measurements were carried out with three-electrode Swagelok-type cells. The working electrode was prepared with $80 \mathrm{wt}$.\% active materials, 10 wt.\% Super P and 10 wt.\% polyvinylidene difluoride (PVDF), and lithium metal was used as counter and reference electrodes. $1 \mathrm{M} \mathrm{LiPF}_{6}$ in ethylene carbonate $(\mathrm{EC})$ and dimethyl carbonate (DMC) $(1: 1, \mathrm{v} / \mathrm{v})$ was used as the electrolyte. The electrode is made of a thin layer of a mixture of anatase $\left(2.5 \mathrm{mg} / \mathrm{cm}^{2}\right)$ and carbon $\left(0.32 \mathrm{mg} / \mathrm{cm}^{2}\right)$ nanocrystals with PVDF $\left(0.32 \mathrm{mg} / \mathrm{cm}^{2}\right)$. The theoretical capacity of the material is $3.02 \mathrm{C} / \mathrm{cm}^{2}$. Cyclic voltammetry was performed at

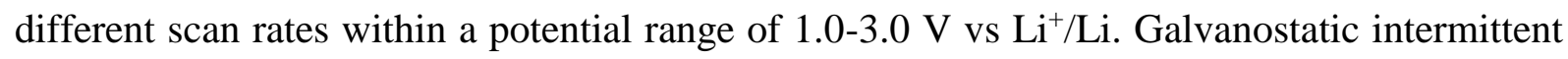
titrations were carried out after one galvanostatic discharge/charge cycle, a constant-current discharge pulse of $84 \mu \mathrm{A} \mathrm{cm}^{-2}$ was applied for 20 minutes, followed by a relaxation duration of $20 \mathrm{~h}$; the current pulse corresponds to a capacity fraction of $1 / 30$. 


\section{Results and discussion}

Transport equations. A specific advantage of electrochemical techniques is to provide an easy measurement of the reaction flux, $J$, at the electrode surface and of the concentration, $c$, of the active species during the electrochemical process. The flux at the electrode surface is given by the current density, $i$

$J^{\circ}=-\frac{i}{n F}$

For an ideal and reversible reaction, $O x+n \mathrm{e}^{-} \rightarrow \operatorname{Red}\left(\right.$ such as, $\mathrm{Li}^{+}+\mathrm{e}^{-} \rightarrow \mathrm{Li}$ ), the concentration, $c$, of the Red species is deduced from the electrode potential from the Nernst law, the concentration of $O x$ species is assumed to be constant,

$c=\exp \left(\frac{n F}{R T}\left(E^{\circ}-E\right)\right)$

where, $E^{0}=R T / n F \times \ln c^{0} ; E$ is the electrode potential vs the pure Red species, $c^{0}$ is the concentration of pure Red.

However, most systems exhibit large deviations from ideality; the Nernst equation gives access to the activity, $a$, of the electroactive species which is linked to the concentration by a factor $f$ called the activity coefficient $f$ :

$a=f c=\exp \left(\frac{n F}{R T}\left(E^{\circ}-E\right)\right)$

The activity coefficient demarcates the equilibrium (thermodynamic) response under concentrated conditions. The intercalation electrodes represent very high concentrations (of the order of $50 \mathrm{M}$ ) that the activity coefficient becomes a strong function of concentration. Various theoretically consistent treatments were used to recover activity coefficient from the open circuit potential (OCP) curves $^{20}$. Here we refer to a previous work dealing with the system Li$\mathrm{V}_{2} \mathrm{O}_{5}$ which exhibits various phase transformations ${ }^{21}$. It was shown ${ }^{22}$ that the large changes in the activity factor vs the concentration were correctly described by the series:

$\ln f=\sum_{j=1}^{n} 0.5\left(\ln f_{j}-\ln f_{j-1}\right) \operatorname{erfc}\left\{\psi_{j}\left(c-c_{T j}\right)\right\}$

$\ln f_{j}$ is the activity factor corresponding to the plateaus of the potential curves; since the reference is the pure compound, $\ln f_{0}=0$; the complementary error function, erfc, was used to describe the change from one factor to the next, $c_{T j}$ is the concentration at the inflexion points of the 
potential curve. $\psi_{i}$ is the steepness of the change of the activity factor around the concentration $c_{T j}$. The various parameters $\ln f_{i}$ and $\psi_{i}$ were adjusted to obtain a suitable representation of the OCP curves. The driving force for diffusion is the gradient of the chemical potential $\mu=\mu^{\circ}+$ $R T \ln f c$; the flux, $J$, of the electroactive species inside the electrode matrix writes:

$J=-\widetilde{D}\left(1+c \frac{\partial \ln f}{\partial c}\right) \frac{\partial c}{\partial x}$

where $\widetilde{D}$ is the component diffusion of the active species, it is related to the chemical diffusion coefficient, $D$, by the equation ${ }^{23,24}$

$D=\widetilde{D}\left(1+\frac{\partial \ln f}{\partial \ln c}\right)$

As pointed out by Levi et al. ${ }^{25}$ this expression may be modified according to the thermodynamic model; to examine the influence of the gradient of the activity coefficient a damping factor, $\varphi$, is introduced in the calculation

$D=\widetilde{D}\left(1+\varphi \frac{\partial \ln f}{\partial \ln c}\right)$

The term $\Gamma$ is called enhancement factor

$\Gamma=1+\varphi \frac{\partial \ln f}{\partial \ln c}$

In a non-stationary state the concentration changes obey the mass balance equation

$$
\frac{\partial c}{\partial t}=-\nabla J=\frac{\partial}{\partial x}\left(D \frac{\partial c}{\partial x}\right)
$$

The integration of transport equations has been solved for different boundary conditions corresponding to galvanostatic or potentiostatic regimes. For a constant value of $D$ algebraic equations are available in literature to interpret the results obtained from transient electrochemical techniques ${ }^{26}$.

However, in nanoparticles the transport occurs over a few atomic layers and the specificity of the motion in this region must be taken into account. Indeed, in the interfacial domain, the looseness of the crystal lattice enlarges the mass transport facilities. In other words it is possible to consider that over these short distances interfacial energy affects the dynamic response. Thus, as a first approximation, it can be considered that the intercalation of ions in the very first atomic layers is extremely rapid and corresponds to an accumulation process occurring instantaneously over a distance $\delta$. This analysis is in good agreement with the concept of relevant surface 
activity thickness (RSAT) introduced by Verbrugge and Tobias ${ }^{27}$ to calculate by electrochemical techniques the surface activity of metallic alloys.

From a dynamic point of view, this approach amounts to considering that, in this film of thickness $\delta$, the coefficient of diffusion $D$ has an infinite value. Of course, within the material, the diffusion coefficient has a constant finite value which is usually the only one taken into consideration; indeed, generally the diffusion phenomena last long enough and affect the deep mass of the material; the amount of material involved in the surface film becomes negligible. To refine our model it was considered that in the few atomic layers in the vicinity of the storage layer, of thickness $\delta$, the diffusion coefficient evolves to reach the classical value of the coefficient in the mass of the material. Here, as in our previous articles ${ }^{16,28}$, it was assumed that $D$ obeys the equation

$D=D^{*} \exp \left(\delta^{\prime} / x\right)$

where $D^{*}$ is the bulk diffusion coefficient and $\delta^{\prime}$ is a characteristic distance called relevant rapid diffusion thickness (RRDT). A diagram is proposed to illustrate the evolution of transport in the surface layers (Fig. 1).

The current density is linked to the flux at the electrode surface $J^{o}$ (Eq. 1). Now, the accumulation process at the interface has to be taken into account

$J^{o}=\delta \frac{\partial c_{0}}{\partial t}-D\left(\frac{\partial c}{\partial x}\right)_{x=\delta}$

$c_{0}$ is the concentration in the surface layer of thickness $\delta$. The introduction of metal ions in the matrix of the crystal creates a volume change of the electrode deduced from the partial molar volume of the electroactive component $\bar{V}_{M}$. It results that during the electrolysis the interface moves with a velocity $\mathrm{v}$

$\mathrm{v}=J \bar{V}_{M}$

The quantity $\bar{V}_{M}$ takes account of the constraints introduced by the insertion of the active element $M$ into the substrate. In general $\bar{V}_{M}$ is clearly lower than the molar volume $V_{M}$; under the action of strong stresses, $\bar{V}_{M}$ can even become negative.

The displacement of the interface is introduced in the diffusion equation which becomes 
$225 D$ depends on the distance, $x$, (Eq. 10) and, according to Eq. 9, the concentration change obeys 226 the equation

$\frac{\partial c}{\partial t}=-\nabla J=D \frac{\partial^{2} c}{\partial x^{2}}-D^{*} \frac{\delta^{\prime}}{x^{2}} \exp \left(\frac{\delta^{\prime}}{x}\right) \frac{\partial c}{\partial x}-\mathrm{v} \frac{\partial c}{\partial x}$

$\frac{\partial^{2} c}{\partial x^{2}}=\frac{c(x+\Delta x, t)-2 c(x, t)+c(x-\Delta x, t)}{(\Delta x)^{2}}$

It must be pointed out that the stability conditions of the finite difference calculation require

However, in contrast with the classical treatment, it is no more possible to integrate the complete diffusion equation into an algebraic form. It is proposed to solve the problem by numerical integration of the diffusion equation according to a procedure already developed to study the electrowinning of refractory metals ${ }^{28}$. The technique requires an analytical representation of the thermodynamic and kinetic properties of the system.

Integration of the diffusion equations. The digital simulation technique used to calculate the transient curve consists in solving the partial differential equation by numerical calculation ${ }^{17}$. A numerical model is set up within a digital computer and the model is allowed to evolve by a set of algebraic laws deriving from equations defining the mass transport. The space ( $x$ coordinate) is divided in small intervals, $\Delta x$, and the time into small time steps, $\Delta t$. The concentration of the electroactive reagent at the electrode surface, at the time $t$, is given by the Nernst equation, and the current density is related to the concentration gradient at the electrode surface

$J^{o}=\delta \frac{c(0, t)-c(0, t-\Delta t)}{\Delta t}-D \frac{c(0, t)-c(\Delta x, t)}{\Delta x}+\mathrm{v} c(0, t)$

$c(\Delta x, t)$ is the concentration at the time $t$ at the distance $\Delta x$ from the electrode surface. $D$ obeys Eq. 10. Concentrations as a function of time and concentration profiles in the bulk of the substrate were calculated from the diffusion equation (Eq. 14). In finite difference scheme the continuous functions are finitized with centered finite scheme for space derivative:

$$
\frac{\partial c}{\partial x}=\frac{c(x+\Delta x, t)-c(x-\Delta x, t)}{2 \Delta x}
$$

that the space interval $\Delta x$ be greater than the diffusion path during the time step: $\Delta x>\sqrt{2 D \Delta t}$; but, on the other hand, to correctly integrate the evolution of the diffusion coefficient (Eq. 10), it is necessary that the space interval, $\Delta x$, be less than the length RRDT, $\delta^{\prime}>\Delta x$. For large time 
intervals, such as in voltammetry at low sweep rate, these conditions may be difficult to meet. More details concerning the calculation procedure can be found in previous papers ${ }^{17,21}$.

\section{Application to lithium insertion in anatase nanocrystals}

Electrode structure - Anatase $\mathrm{TiO}_{2}$ has been extensively studied over the past decade due to its application as electrode material for $\mathrm{Li}$ batteries $^{29,30}$. In a simplifying assumption and in agreement with the known structure of the nanoparticles, the electrode material will be considered as a collection of nano-platelets of parallelepiped shape ${ }^{31}$ (Fig. 2) in contact with the electrolyte and with the carbon powder which provides the electric contact. For an electrode containing $m$ gram of active compounds per unit surface the number of particles is

$n_{p}=m /(\rho \times a \times a \times b)$

where $\rho$ is the specific mass of the compound. It is assumed that the lithium diffusion occurs only in one direction perpendicular to the surface $a \times a .^{31}$. The real active surface for the electrochemical reaction is

$S_{p}=n_{p} \times a \times a$

The electrochemical reaction occurring in the battery is described by the reaction

$\mathrm{TiO}_{2}+y \mathrm{Li}^{+}+y \mathrm{e}^{-} \leftrightarrow \mathrm{Li}_{y} \mathrm{TiO}_{2}$

where $y$ is the lithium intercalation ratio. Upon lithiation to $\mathrm{Li}_{0.5} \mathrm{TiO}_{2}$, anatase is observed to undergo a tetragonal to orthorhombic phase transition ${ }^{32}$. The thermodynamically stable ordered phase at $y=0.5$ can accommodate excess $\mathrm{Li}$ in vacant sites up to $y=0.6^{33}$; in agreement with the model developed above, it was shown that the diffusion in this phase is strictly one dimensional ${ }^{31}$. In order to improve the storage capacity of the electrode, fluorinated anatase crystals were also investigated. The samples used in the present description correspond to the fluorinated anatase, $\mathrm{Ti}_{0.78} \square_{0.22} \mathrm{O}_{1.12} \mathrm{~F}_{0.40}(\mathrm{OH})_{0.48}$, as described in previous papers ${ }^{19,34}$.

An estimate of the particle sizes is deduced from microscopic investigation showing that the anatase grains have a dimension of the order of ten nanometers ${ }^{19,35}$. In our study, as a first approximation, they will be assimilated to parallelepipeds according to the representation of Fig.2, with, for dimension: $a=3 \mathrm{~nm}$ and $b=12 \mathrm{~nm}$. 
According to the synthesis of the electrodes, the cathodic layer has a thickness of $10 \mu \mathrm{m}$. In this layer the weight of anatase or fluorinated anatase unit is $2.5 \mathrm{mg} / \mathrm{cm}^{2}$; the number of particles, $n_{p}$, and their active electrochemical area, $S_{p}$, deduced from Eqs 18, 19 are reported in Table 1.

Electrochemical analysis - The electrode potential, E (vs pure lithium metal), corresponding to the electrochemical reaction (Eq. 20) is given by the Nernst's equation

$E=E^{\circ}-\frac{R T}{F} \ln f_{L i} c_{L i}$

with

$E^{\circ}=\frac{R T}{F} \ln c_{L i}^{o}$

$c_{L i}^{o}$ is the concentration of pure lithium metal. The index $y$ (Eq. 20) and $c_{L i}$ are linked through the equation

$c_{L i}=y / V_{L_{y} \mathrm{TiO}_{2}}$

$V_{L i_{y} \mathrm{TiO}_{2}}$ is the molar volume of the compound

$V_{\mathrm{Li}_{y} \mathrm{TiO}_{2}}=V_{\mathrm{TiO}_{2}}+y \bar{V}_{\mathrm{Li}}$

$\bar{V}_{\mathrm{Li}}$ is the partial molar volume of lithium. The above relation assumes that $\bar{V}_{\mathrm{Li}}$ remains constant in the concentration range studied; however, the imprecision eventually introduced is very small; indeed, as indicated above, $\bar{V}_{\mathrm{Li}}$ is much smaller than the molar volume of lithium $\left(\bar{V}_{\mathrm{Li}} \approx\right.$ $\left.0.1 V_{L i}\right)$. As a result, the variations in volume of the substrate during the insertion of lithium are always small.

The value of the activity coefficient, $f_{L i}$, is deduced from the Eq. 4 ; the parameters, $f_{j}$ and $\psi_{j}$, the index $j$ varying from 1 to 4 , are adjusted in order to obtain a good fit between the calculated curve and experimental OCP curve (Fig. 3). The goodness-of fit values of the parameters involved in the calculation are shown in Table 2.

Analysis by cyclic voltammetry, pseudo-capacitive contribution - The cyclic voltammograms on anatase, or fluorinated anatase $\mathrm{Ti}_{0.78} \square_{0.22} \mathrm{O}_{1.12} \mathrm{~F}_{0.4} \mathrm{OH}_{0.45}$ particles are reported in Figs 4-5.

To analyze the voltammograms it was assumed that the electrochemical response depends of two components ${ }^{36}$ : the faradaic current, $i_{F}$, coming from the lithium insertion process and from 
the charge-transfer process with surface atoms (pseudo-capacity), and the non-faradaic current, $i_{C}$, arising from double layer capacitance, which corresponds, in our field of study, to a very small current ${ }^{37}$; it can be neglected ${ }^{36}$. In the literature it is generally accepted that the faradaic contribution is the sum of two terms ${ }^{36,38}$ :

$i_{F}=k_{1} v^{1 / 2}+k_{2} v$

where $v$ is the scan rate; $k_{1}$ and $k_{2}$ are two constants, the first term arises from the diffusion controlled insertion, and the second one from the pseudo-capacitive effect.

It is now proposed to interpret the cyclic voltammetry response in a more precise way by taking into account the characteristic features of atomic-scale transport.

Surface transport and capacitive effect - The pseudo-capacitive behavior arises from the transport properties in the first atomic layers and from the fast storage of the active material at the surface of nanoparticles. These effects come from the properties of the interface at atomic scale which result in a variation of the diffusion velocity (Eq. 10) and in the existence of an instantaneously charged layer of thickness $\delta$ (RSAT).

The finite differences technique is used to calculate the current response during a cyclic potential scan rate corresponding to lithium insertion (Figs 4-5). The morphological change of the crystal lattice due to the lithium insertion induces a change of the thicknesses $\delta$ and $\delta$ '. It is assumed that these values reach a maximum for $c=c_{p}$; for anatase: $c_{p}=15.9 \times 10^{-3} \mathrm{~mol} / \mathrm{cm}^{3}$, for fluorinated anatase: $c_{p}=12.3 \times 10^{-3} \mathrm{~mol} / \mathrm{cm}^{3}$. The corresponding intercalation ratios (Eq. 20) are respectively: $y=0.34$ and $y=0.26$. To make it easier to implement the calculation, it was assumed that $\delta$ and $\delta$ ' obey the equations

$\delta=\delta_{\max } \exp -\frac{\left(c-c_{p}\right)^{2}}{3 c_{p}^{2}}$

and

$\delta^{\prime}=0.25 * \delta$

The two above empirical relations, and the values of $D^{*}$ and $\delta_{\max }$ are adjusted in order to obtain the best fit with the experimental curves (Table 3). It should be noted that the $\delta_{\max }$ values are of the same order of magnitude as those obtained during the electrochemical preparation of metal alloys $^{28,39}$ (a few nanometers). 
345 The storage process can be interpreted in terms of pseudo capacitance, $C$. The charge density $q$, stored in the RSAT layer is

$348 c(0)$ is the concentration at the electrode surface at a potential, $E$. The value of the capacitance, $349 C$, is deduced from the classical formula, $C=d q / d E$. The charge increment, $d q$, induced by a potential increment, $d E$, can be deduced from concentration change, $d c(0)$, calculated from the Nernst equation (Eq. 3); then

In Table 3 are reported the maximum values $C_{\max }$ of the capacitance, which occurs when $\delta=$ $\delta_{\max }$, so, according to Eq. 26 , when $c=c_{p}$. The values of the so-called capacitance may seem to be enormous. However, they are in good agreement with the experimental observations. For example, in the fluorinated anatase at a scan rate of $10 \mathrm{mV} / \mathrm{s}$, almost all the current has a capacitive origin (Fig. 6); thus, to obtain a current density, $i_{c}$, of the order of $10 \mathrm{~mA} / \mathrm{cm}^{2}$ with a scan rate, $v$, of $10 \mathrm{mV} / \mathrm{s}$, a capacitive constant, $C$, of the order of one Farad is required (reminder of the formula: $i_{c}=\mathrm{C} \times v$ ).

Due to the large increase in the electroactive surface it has sometimes been suggested that the double-layer capacity could account for the large values obtained. This suggestion is hardly conceivable: for usual solvents, the order of magnitude of the double-layer capacity ${ }^{40}$ is at most around $18 \mu \mathrm{F} / \mathrm{cm}^{2}$, which corresponds, even for an area of around $550 \mathrm{~cm}^{2}$, to a capacity of 10 $\mathrm{mF}$, a value much lower than those obtained experimentally (Table 3). For that matter, a large part of this paper has been written in an attempt to analyze this phenomenon. The importance of the so-called capacitive response is also underlined by the work of Wang et al. ${ }^{36}$ which show that, even at low scan rates $(0.5 \mathrm{mV} / \mathrm{s})$, the capacitive response is not negligible.

This model also makes it possible to link the variations of the so-called capacity to the size of the particles. Indeed, the capacitive effect depends directly on the contact surface, $S_{p}$, which is inversely proportional to the particle size as can be deduced from Eqs 18, 19. This suggests that the capacitive effect will also be inversely proportional to the particle size. This dependence is well observed experimentally ${ }^{36}$. At lower sweep rates the influence of the capacitive current decreases, concentration profiles indicate a deeper penetration of lithium atoms into the bulk of 
the electro material, the electrochemical response is getting closer to the classical laws of diffusion.

Diffusion and thermodynamics - In order to obtain a more accurate determination of the diffusion into the bulk of anatase particles dependence a galvanostatic intermittent titration technique is used. The procedure consists of a series of current pulses, each followed by a relaxation time, in which no current passes through the cell. The digital simulation technique is used to calculate the electrode response from the model presented above. As deduced from the cyclic voltammetry investigations the surface storage capacity is taken into account and the bulk diffusion coefficient is adjusted to obtain the best fit with the experimental results. As shown in Fig. 7, the fitted curves correctly represent the experimental curves. The values of the diffusion coefficients are shown in Fig. 8.

It should be noted that wide fluctuations in the diffusion coefficient are obtained as a function of the degree of lithium insertion. These variations reflect the change in structure of the anatase crystals; they can result in a change in the thermodynamic properties of the system that is reflected in the evolution of the enhancement factor (Eq. 7). The curves of Fig. 8 show that there is some correlation between the evolution of this factor and that of diffusion coefficients. This approach makes it possible to envisage a reasoned study linking the structural, thermodynamic and kinetic properties of the system. The anatase OCP curve (Fig. 3) exhibits two potential plateaus at $E_{1}=1.808 \mathrm{~V}$, and $E_{2}=1.605 \mathrm{~V}$, which correspond respectively to the compounds $\mathrm{TiO}_{2} \mathrm{Li}_{\mathrm{y}}$, with $y_{1}=0.302$ and $y_{2}=0.635$. The Gibbs energies of lithium insertion in the compounds are $\Delta \mathrm{G}_{1}=-52.7 \mathrm{~kJ}$ and $\Delta \mathrm{G}_{2}=-98.4 \mathrm{~kJ}$. The formation of these compounds leads to a large departure from ideality during the insertion of lithium. This results in significant variations in the activity coefficient, $f$, which according to Eq. 5, influences the diffusional behavior of lithium.

\section{Conclusion}

The analysis of lithium insertion, in a host material made of nanoparticles shows that special attention needs to be given to the study of transport phenomena in the first atomic layers of the interface. In this region the looseness of the crystal lattice enlarges the mass transport facilities. It results in additional effects not included in the classical analyses. To describe the system behavior it is required to introduce the characteristics of surface transport in the diffusion 
equation which can be solved by numerical calculation. This model takes into account the actual contact surface and includes the concept of storage layer correlated with the increase of the diffusion coefficient in the vicinity of the interface. In addition, the variations of transport kinetics are related to the thermodynamic properties of the medium.

This work highlights the primary and complex role of atomic-scale transport phenomena in the surface layers of electrode materials. The analysis thus developed can be a useful tool for directing research towards structure with rapid charge kinetics and thus increase the available power. This is how the idea of fluorination of anatase came about: this treatment improves diffusivity, surface storage and pseudo-capacitance (Table 3). In addition, the study focuses directly on the capacitive properties of the electrode; it can thus be useful for the research, development and processing of materials for super-capacitors

The significant effect of transport in the first atomic layers of the substrate is highlighted. To facilitate the implementation of the model, simplifying assumptions were arbitrarily introduced: shape of particles, variation of the thickness of the storage layers (Eqs 26, 27); in addition, the finite difference treatment could be refined. Nevertheless, this study can be adapted and may provide a useful basis for more accurate analysis of the electrochemistry of nanoparticle systems. In the present work we have endeavored to develop a method of analysis of these mechanisms. The theoretical procedure is illustrated on the model case of lithium insertion in anatase nanoparticles studied by cyclic voltammetry or by galvanostatic titration; in particular, it accounts for the high values of the observed capacitive effects. Moreover, it can be easily extended to the analysis of charge-discharge processes under various experimental conditions.

\section{Acknowledgement}

The research leading to these results has received funding from the French National Research Agency under Idex@Sorbonne University for the Future Investments program (No. ANR-11IDEX-0004-02) and the Nature Science Foundation of China (No. 21805209).

\section{References}


444

445

446

447

448

449

450

451

452

453

454

455

456

457

458

459

460

461

462

463

464

465

466

467

468

469

470

471

472

473

474

475

476

477

478

1. M. Yoshio, R. J. Brodd, A. Kozawa, Lithium batteries, Springer, New-York (2009), doi: 10.1007/978-0-387-34445-4

2. R.Van Noorden, Nature, 507, 26-28 (2014), doi: 10.1038/507026a.

3. E. Samadani, M. Mastali, S. Farhad et al., Int. J. Energy Res., 40, 379-392 (2016), doi: 10.1002/er.3378.

4. J. B. Goodenough and Y. Kim, Chem. Mater., 22, 587-603 (2010), doi: $10.1021 / \mathrm{cm} 901452 \mathrm{z}$.

5. A. S. Aricò, P. Bruce, B. Scrosati, J.-M. Tarascon, and W. van Schalkwijk, Nature Materials, 4, 366-377 (2005), doi: 10.1038/nmat1368.

6. R. Malik, F. Zhou and G. Ceder, Nature Materials, 10, 587-590 (2011), doi: 10.1038/nmat3065.

7. D. Cogswell and M. Bazant, ACS Nano, 6, 2215-2225 (2012), doi: 10.1021/nn204177u.

8. G. Oyama, Y. Yamada, R. Natsui, S. Nishimura and A. Yamada, J. Phys. Chem., 116, 7306-7311 (2012), doi : org/10.1021/jp300085n

9. L. R. De Jesus, P. Stein, J. Andrews, Y. Luo, B.-Xiang Xu and S. Banerjee, Materials Horizons, 5, 486-498 (2018), doi: 10.1039/c8mh00037a.

10. K. Gallagher, D. Dees, A. Jansen, D. Abraham and S. Kang., J. Electrochem. Soc., 159, A2029-A2037 (2012), doi: 10.1149/2.015301jes.

11. D. Baker and M. Verbrugge, J. Electrochem. Soc., 159, A1341-1350 (2012), doi:

10.1149/2.002208jes.

12. D. Bernardi, R. Chandrasekaran and J. Young Go, J. Electrochem. Soc., 160, A1430A1441 (2013), doi: 10.1149/2.042309jes.

13. A. Mistry, F. Cano-Banda, D. Law, A. Hernandez-Guerrero and P. Mukerjee, J. Mater. Chem., 7, 8882-8888 (2019), doi: 10.1039/c9ta01339c.

14. L. Kavan, J. Rathouský, M. Grätzel, V. Shklover, and A. Zukal, J. Phys. Chem. B, 104, 12012-12020 (2000), doi: 10.1016/S0167-2991(03)80455-X.

15. D. Fattakhova, L. Kavan, and P. Krtil, J. Solid State Electrochem, 5, 196-204 (2001), doi: $10.1007 / \mathrm{s} 100080000138$.

16. F. Lantelme and M. Chemla, Journal of Electroanal. Chem., 396, 203-209 (1995) doi: 10.1016/0022-0728(95)03933-8.

17. F. Lantelme, Computer Simulation of Electrochemical Processes, p.180 in Q. Zhiyu, X. Zhihong and L. Honglin (eds), Computerized Physical Chemistry of Metallurgy and Materials, The Metallurgical Industry Press, (1999) Beijing.

18. D. Britz, Digital Simulation in Electrochemistry, Springer-Verlag, Berlin Heidelberg, (1981), doi: 10.1007/978-3-662-21819-8. 
19. W. Li, D. Corradini, M. Body, C. Legein, M. Salanne, J. Ma, K. W. Chapman, P. J. Chupas, A.-L. Rollet, C. Julien, K. Zhagib, M. Duttine, A. Demourgues, H. Groult, and D. Dambournet, Chem. Mater., 27, 5014-5019 (2015), doi: 10.1021/acs.chemmater.5b01407.

20. S. Srinivasan and J. Newman, J. Electrochem. Soc., 151, A1530-A1538 (2004), doi: $10.1149 / 1.1785012$.

21. F. Lantelme, A. Mantoux, H. Groult, and D. Lincot, J. Electrochem. Soc., 150, A1202A1208 (2003), doi:10.1149/1.1595658.

22. F. Lantelme, A. Mantoux, H. Groult, and D. Lincot, Solid State Ionics, 177, 205-209 (2006), doi: 10.1016/j.ssi.2005.10.024.

23. L. S. Darken, Trans. Am. Inst. Mining Metall. Eng., 175, 184 (1948).

24. F. Lantelme, H. Groult, and N. Kumagai, Electrochim. Acta, 45, 3171-3180 (2000), doi: 10.1016/S0013-4686(00)00474-6.

25. M. D. Levi, and D. Aurbach, J. Phys. Chem. B, 101, 4630-4640 (1997), doi: 10.1021/jp9701909.

26. J. Heinze, Berichte der Bunsengesellschaft für physikalische Chemie, 85, 1085-1086 (1981), doi: 10.1002/bbpc.19810851204.

27. M. W. Verbrugge and C. W. Tobias, J. Electrochem. Soc., 132, 1298-1307 (1985), doi: $10.1149 / 1.2114106$.

28. F. Lantelme and A. Salmi, Journal of Physics and Chemistry of Solids, 57, 1555-1565 (1996), doi: 10.1016/0022-3697(96)00061-3.

29. T. Ohzuku, T. Kodama, and T. Hirai, Journal of Power Sources, 14, 153-166 (1985), doi: 10.1016/0378-7753(85)88026-5.

30. S. Y. Huang, L. Kavan, I. Exnar, and M. Grätzel, J. Electrochem. Soc., 142, L142-L144 (1995), doi: 10.1149/1.2048726.

31. A. A. Belak, Y. Wang, and A. Van der Ven, Chem. Mater., 24, 2894-2898 (2012), doi: $10.1021 / \mathrm{cm} 300881 \mathrm{t}$.

32. M. Wagemaker, W. J. H. Borghols, and F. M. Mulder, J. Am. Chem. Soc., 129, 43234327 (2007), doi: 10.1021/ja067733p.

33. K. Shen, H. Chen, F. Klaver, F. M. Mulder, and M. Wagemaker, Chem. Mater., 26, 16081615 (2014), doi: 10.1021/cm4037346.

34. J. Ma, W. Li, B. J. Morgan, J. Światowska, R. Baddour-Hadjean, M. Body, C. Legein, O. J. Borkiewicz, S. Leclerc, .H. Groult, F. Lantelme, C. Laberty-Robert, and D. Dambournet, Chem. Mater., 30, 3078-3089 (2018), doi : 10.1021/acs.chemmater.8b00925.

35. W. Li, M. Body, C. Legein, O. J. Borkiewicz, and D. Dambournet, Eur. J. Inorg. Chem., 2017, 192-197 (2017), doi: 10.1002/ejic.201601160.

36. J. Wang, J. Polleux, J. Lim, and B. Dunn, J. Phys. Chem. C, 111, 14925-14931 (2007), doi: 10.1021/jp074464w. 
516 37. B. E. Conway, V. Birss, and J. Wojtowicz, J. Power Sources, 66, 1-14 (1997), doi:

$517 \quad 10.1016 / \mathrm{S} 0378-7753(96) 02474-3$.

518 38. T. Brezesinski, J. Wang, J. Polleux, B. Dunn, and S. H. Tolbert, J. Am. Chem. Soc., 131, 519 1802-1809 (2009), doi: 10.1021/ja8057309.

520 39. A. Salmi, Y. Berghoute, and F. Lantelme, Electrochim. Acta, 40, 403-411 (1995), doi:

521 10.1016/0013-4686(94)00288-C.

522 40. S. Srinivasan, Fuel cells. From Fundamental to Applications, Ch. 2, Springer (2006), doi: 523 10.1007/0-387-35402-6. 


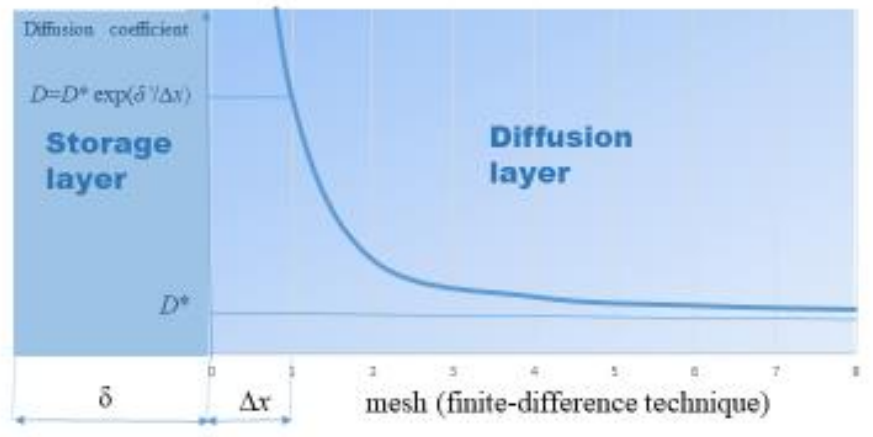

527

528 Figure 1. Diagram illustrating the transport in surface layers.

529

530

531

532

533

534

535

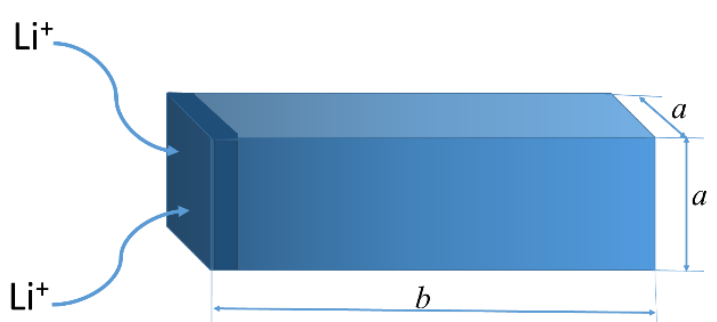

536 Figure 2. Platelet-like nano-particle ${ }^{31}$, simplified form used for the calculation. 


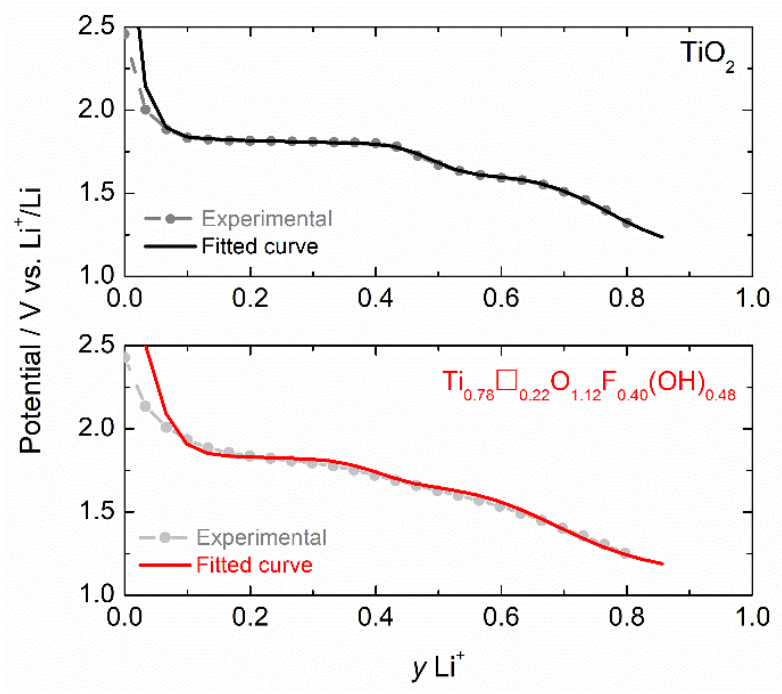

539 Figure 3. Open circuit potential curve, anatase and fluorinated anatase $\left(y \mathrm{Li}^{+}\right)$is the intercalation 540 ratio).

541

542

543

544

545

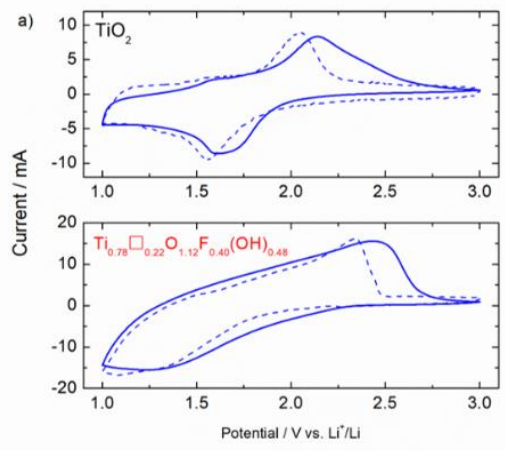

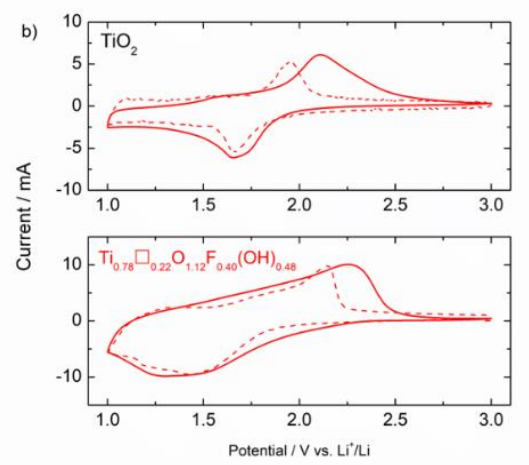

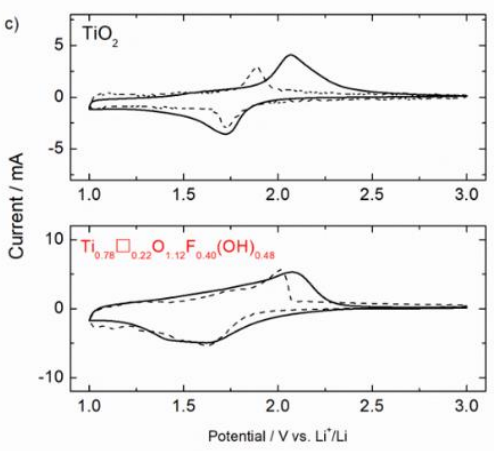

Figure 4. Experimental (continuous line) and fitted (dashed line) cyclic voltammograms of

547 lithium insertion/deinsertion in anatase and fluorinated anatase; electrode surface: $0.78 \mathrm{~cm}^{2}$.

548 Sweep rates: (a) $v=10 \mathrm{mV} \mathrm{s}^{-1}$, (b) $v=5 \mathrm{mV} \mathrm{s}^{-1}$, (c) $v=2 \mathrm{mV} \mathrm{s}^{-1}$. 


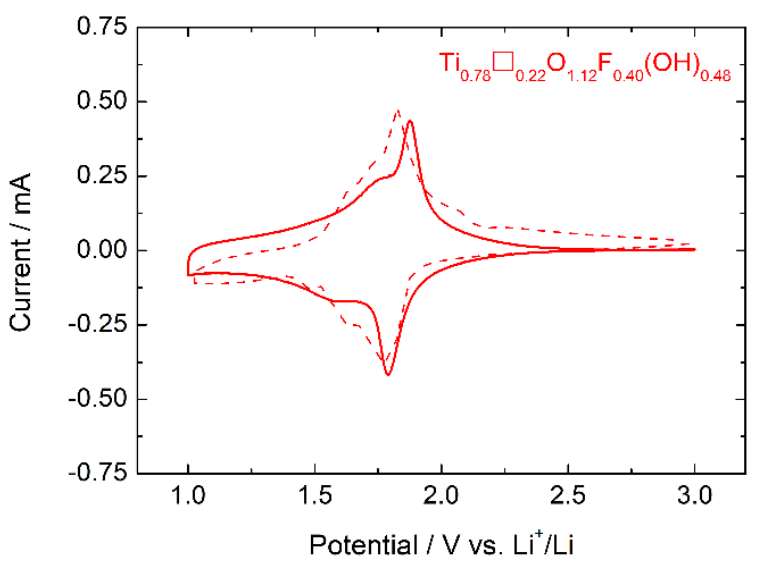

551

552 Figure 5. Experimental (continuous line, after 2 cycles activation) and fitted (dashed line) cyclic voltammograms of lithium insertion/deinsertion in fluorinated anatase; electrode surface: $0.78 \mathrm{~cm}^{2}$; scan rate: $v=0.05 \mathrm{mV} \mathrm{s}^{-1}$.

555

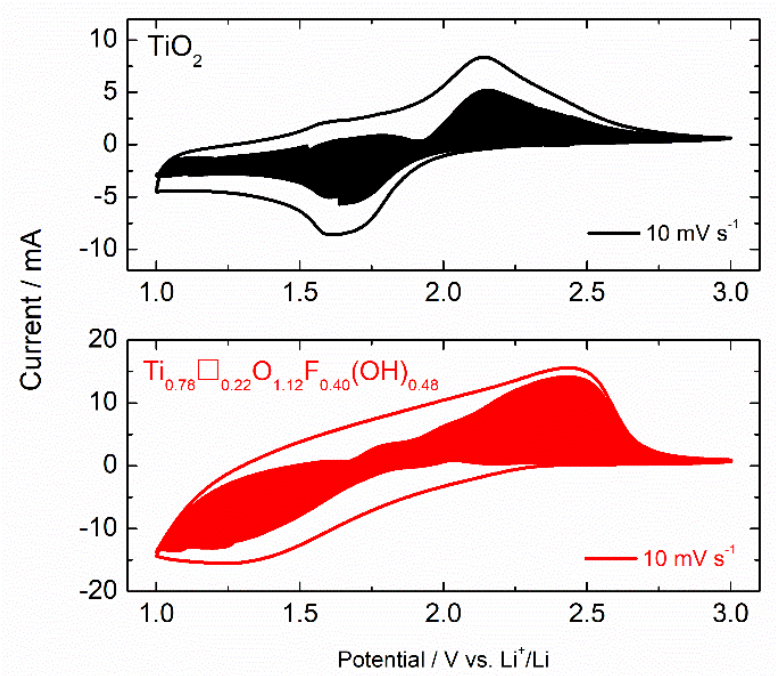

561 Figure 6. Cyclic voltammograms of lithium insertion/deinsertion in anatase and fluorinated 562 anatase; electrode surface: $0.78 \mathrm{~cm}^{2}$; shaded region, capacitive current determined from the 563 procedure using Eq. 25 at $v=10 \mathrm{mV} \mathrm{s}^{-1}$. 

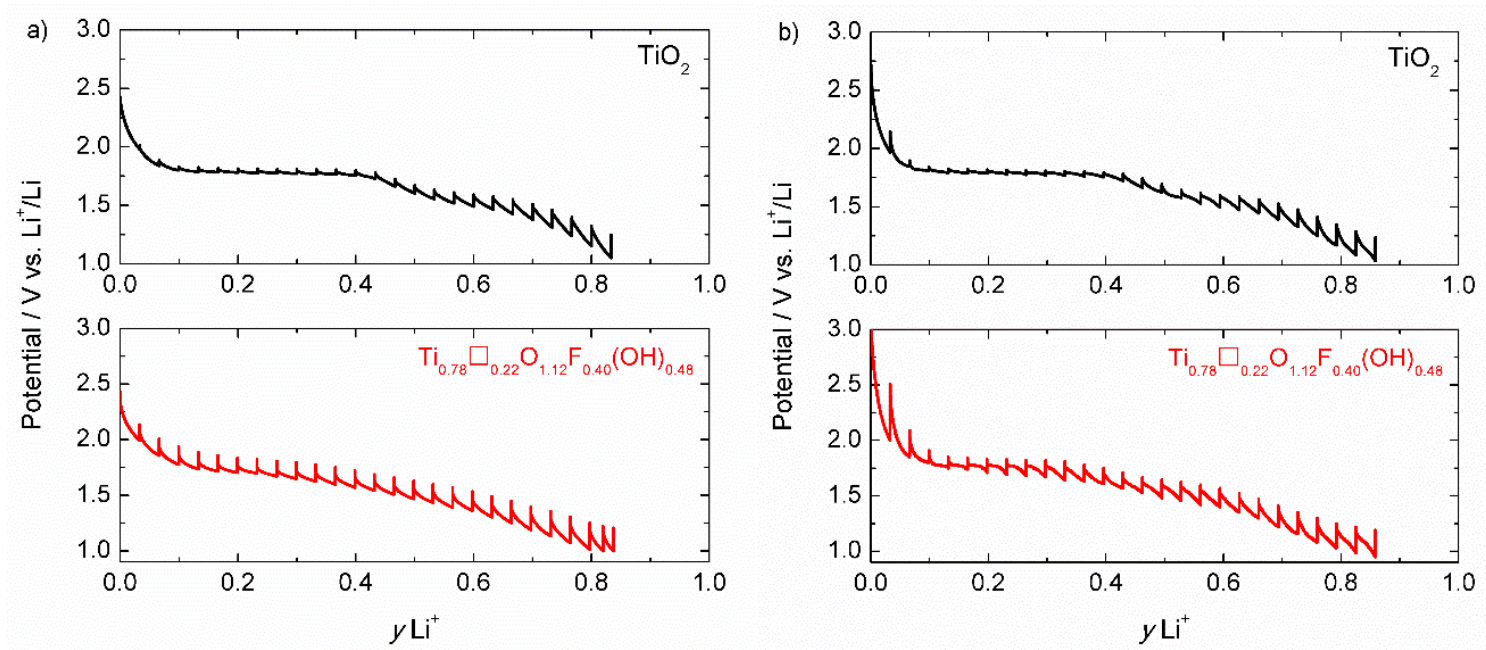

568 Figure 7. Galvanostatic intermittent titration. (a) Experimental and (b) fitted curves in anatase 569 and fluorinated anatase; current density: $84 \mu \mathrm{A} \mathrm{cm}^{-2}$, duration of the galvanostatic charge: 1200 s, relaxation time: $20 \mathrm{~h}\left(y \mathrm{Li}^{+}\right.$is the intercalation ratio).
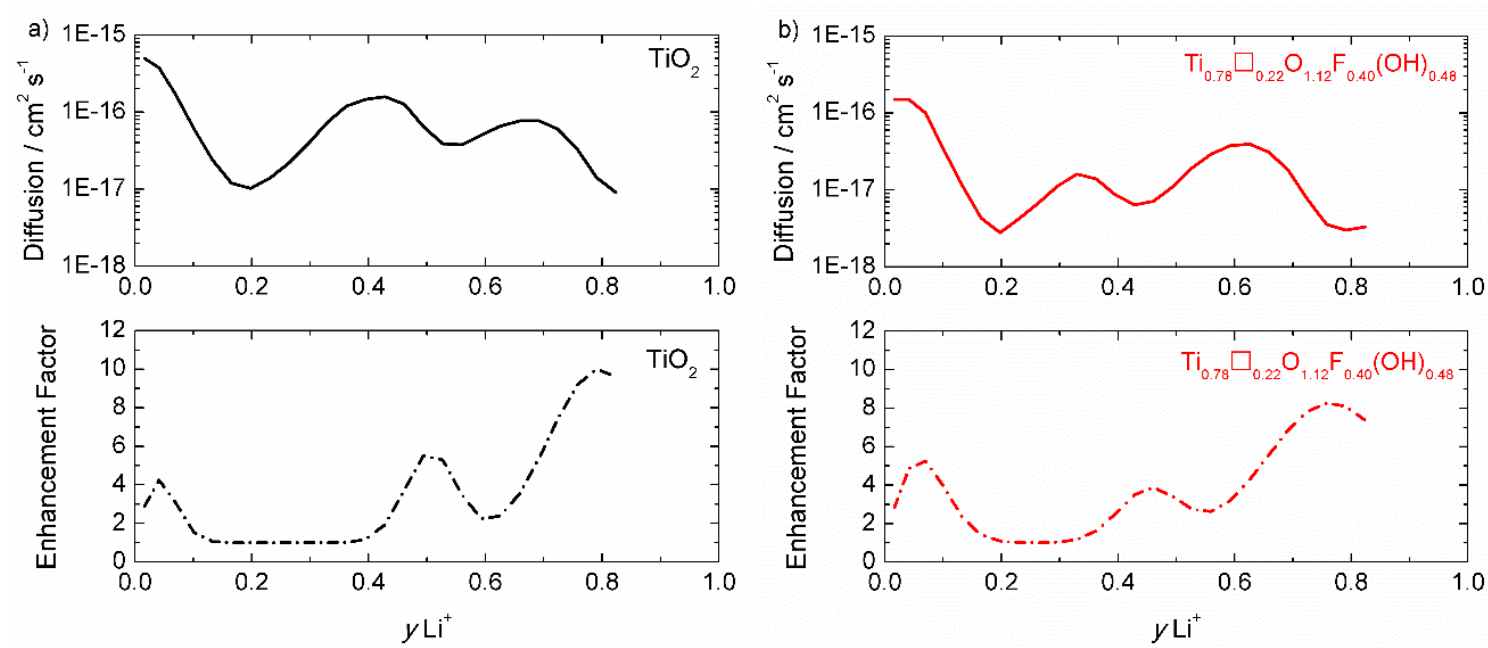

Figure 8. Diffusion coefficient deduced from the different sequence of the galvanostatic

577 intermittent titration curves. Calculated enhancement factors (Eq. 8, with $\varphi=0.26$ ). (a): $\mathrm{TiO}_{2}$;

578 (b): $\mathrm{Ti}_{0.78} \square 0.22 \mathrm{O}_{1.12} \mathrm{~F}_{0.40}(\mathrm{OH})_{0.48}$. 
Table Content

Table 1. Characteristic quantities of the system.

\begin{tabular}{|c|c|c|c|}
\hline \multicolumn{2}{|c|}{$\begin{array}{c}\text { Dimension of } \\
\text { nano-particles }\end{array}$} & \multicolumn{2}{c|}{ For $1 \mathrm{~cm}^{2}$ (electrode surface) } \\
\hline$a(\mathrm{~nm})$ & $b(\mathrm{~nm})$ & $\begin{array}{l}n_{p}, \text { number of } \\
\text { nano-particles }\end{array}$ & $\begin{array}{l}S_{p} \text {, electrochemical } \\
\text { interface },\left(\mathrm{cm}^{2}\right)\end{array}$ \\
\hline 3.0 & 12 & $6.18 \times 10^{15}$ & 556 \\
\hline
\end{tabular}

585

Table 2. Values of the parameters used to describe the evolution of the activity coefficient (Eq. 4).

F-- $\mathrm{TiO}_{2}$ represents $\mathrm{Ti}_{0.78} \square_{0.22} \mathrm{O}_{1.12} \mathrm{~F}_{0.40}(\mathrm{OH})_{0.48}$.

Logarithms of the activity coefficients, $\ln f_{j}$.

589

\begin{tabular}{|l|l|l|l|l|}
\hline & $\ln f_{1}$ & $\ln f_{2}$ & $\ln f_{3}$ & $\ln f_{4}$ \\
\hline $\mathrm{TiO}_{2}$ & -41 & -57 & -63 & -138 \\
\hline $\mathrm{F}^{-\mathrm{TiO}_{2}}$ & -40 & -58 & -63 & -172 \\
\hline
\end{tabular}

590 Concentrations at the transition points, $c_{T j}\left(\mathrm{~mol} \mathrm{~cm}^{-3}\right)$

591

\begin{tabular}{|l|l|l|l|l|}
\hline & $c_{T 1}$ & $c_{T 2}$ & $c_{T 3}$ & $c_{T 4}$ \\
\hline $\mathrm{TiO}_{2}$ & 0.057 & 0.035 & 0.025 & 0.0004 \\
\hline $\mathrm{F}_{-\mathrm{TiO}_{2}}$ & 0.057 & 0.033 & 0.019 & 0.0002 \\
\hline
\end{tabular}

Table 3. Values of the diffusion coefficient at the interface, $D^{*}$ (Eq. 10). Maximum value of the storage thickness, $\delta_{\max }$ (Eq. 26), and pseudo-capacitance associated to that thickness (Eq. 29).

\begin{tabular}{|l|c|c|c|}
\hline & $D^{*}\left(10^{-17} \mathrm{~cm}^{2} \mathrm{~s}^{-1}\right)$ & $\delta_{\max },(\mathrm{nm})$ & $\left.C_{\max }(\mathrm{mF} \mathrm{cm})^{-2}\right)$ \\
\hline $\mathrm{TiO}_{2}$ & 1.3 & 2.0 & 283 \\
\hline $\mathrm{F} \mathrm{TiO}_{2}$ & 4.0 & 4.7 & 978 \\
\hline
\end{tabular}

